\title{
The Philae lander reveals low-strength primitive ice inside cometary boulders
}

https://doi.org/10.1038/s41586-020-2834-3

Received: 7 April 2020

Accepted: 28 August 2020

Published online: 28 October 2020

Check for updates

\author{
Laurence $\mathrm{O}^{\prime}$ Rourke $^{1 凶}$, Philip Heinisch ${ }^{2}$, Jürgen Blum ${ }^{2}$, Sonia Fornasier ${ }^{3,4}$, Gianrico Filacchione $^{5}$, \\ Hong Van Hoang ${ }^{3,6}$, Mauro Ciarniello ${ }^{5}$, Andrea Raponi ${ }^{5}$, Bastian Gundlach ${ }^{2}$, Rafael \\ Andrés Blasco ${ }^{7}$, Björn Grieger ${ }^{8}$, Karl-Heinz Glassmeier ${ }^{2}$, Michael Küppers', Alessandra Rotundi ${ }^{5}{ }^{5}$, \\ Olivier Groussin ${ }^{10}$, Dominique Bockelée-Morvan ${ }^{3}$, Hans-Ulrich Auster ${ }^{2}$, Nilda Oklay", \\ Gerhard Paar ${ }^{12}$, Maria del Pilar Caballo Perucha ${ }^{12}$, Gabor Kovacs ${ }^{13}$, Laurent Jorda ${ }^{10}$, \\ Jean-Baptiste Vincent ${ }^{14}$, Fabrizio Capaccioni ${ }^{5}$, Nicolas Biver ${ }^{3}$, Joel Wm. Parker ${ }^{15}$, \\ Cecilia Tubiana ${ }^{5,16} \&$ Holger Sierks ${ }^{16}$
}

\begin{abstract}
On 12 November 2014, the Philae lander descended towards comet 67P/ChuryumovGerasimenko, bounced twice off the surface, then arrived under an overhanging cliff in the Abydos region. The landing process provided insights into the properties of a cometary nucleus ${ }^{1-3}$. Here we report an investigation of the previously undiscovered site of the second touchdown, where Philae spent almost two minutes of its cross-comet journey, producing four distinct surface contacts on two adjoining cometary boulders. It exposed primitive water ice-that is, water ice from the time of the comet's formation 4.5 billion years ago-in their interiors while travelling through a crevice between the boulders. Our multi-instrument observations made 19 months later found that this water ice, mixed with ubiquitous dark organic-rich material, has a local dust/ice mass ratio of $2.3_{-0.16}^{+0.2}: 1$, matching values previously observed in freshly exposed water ice from outbursts ${ }^{4}$ and water ice in shadow ${ }^{5,6}$. At the end of the crevice, Philae made a 0.25 -metre-deep impression in the boulder ice, providing in situ measurements confirming that primitive ice has a very low compressive strength (less than 12 pascals, softer than freshly fallen light snow) and allowing a key estimation to be made of the porosity ( $75 \pm 7$ per cent) of the boulders' icy interiors. Our results provide constraints for cometary landers seeking access to a volatile-rich ice sample.
\end{abstract}

Fly-bys and rendezvous missions have been central to the provision of close-up images of cometary surface structures, delivering important insights into the chemical and physical processes that have defined them ${ }^{7}$. The presence of boulders on their surfaces with sizes ranging from the metre scale up to tens of metres-often in locations not matching where they were initially exposed-certainly points to the dynamic nature of their creation ${ }^{8,9}$. A determination of mechanical strength properties derived from in situ measurements carried out on the primitive ice located in the interior of a cometary boulder allows unique comparisons to be made with the cometary body internal structure. Furthermore, these properties provide information about the comet's dynamical history and deliver important constraints for the design of cometary landers and cryogenic sample return missions.

The European Space Agency's Rosetta mission ${ }^{10}$ was launched in 2004 and began orbiting comet 67P/Churyumov-Gerasimenko in August 2014. On 12 November 2014, the Philae lander was released with a faulty harpoon system, touching down on the surface on two occasions while also experiencing a glancing collision against the Hatmehit depression edge. After touchdown 2 (TD2), it proceeded to its final position at touchdown 3 (TD3), located under an overhang in the Abydos region of the comet $^{1}$ (see Fig. 1a-d). Although scientific analysis of data ${ }^{1,2,11}$ from Philae's first and third touchdown points have provided important insights into the properties of a cometary nucleus, the location and scientific implications stemming from the second touchdown point were unknown up to now. Its importance was noted ${ }^{12}$, however, as Philae was found to have changed both velocity and rotation rate at this location, as well as having penetrated the surface with the Rosetta Lander Magnetometer and Plasma Monitor ${ }^{13}$ (ROMAP) sensor, possibly exposing ice at the same time.

The production of a new Philae landing trajectory (Supplementary Methods, Supplementary Fig. 1) served to start the search for TD2, with a ridge region identified as being a likely candidate for its

${ }^{1}$ European Space Agency (ESA), European Space Astronomy Centre (ESAC), Madrid, Spain. ${ }^{2}$ Institut für Geophysik und extraterrestrische Physik, Technische Universität Braunschweig, Braunschweig, Germany. ${ }^{3}$ LESIA, Observatoire de Paris, Université PSL, CNRS, Université de Paris, Sorbonne Université, Meudon, France. ${ }^{4}$ Institut Universitaire de France (IUF), Paris, France.

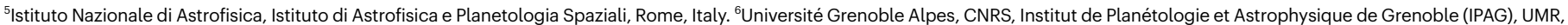
Grenoble, France. ${ }^{7}$ Telespazio Vega UK Ltd for the European Space Agency (ESA), European Space Astronomy Centre (ESAC), Madrid, Spain. ${ }^{8}$ Aurora Technology BV for the European Space Agency (ESA), European Space Astronomy Centre (ESAC), Madrid, Spain. ${ }^{9}$ Dipartimento di Scienze e Tecnologie, Universitá degli Studi di Napoli Parthenope, Naples, Italy. ${ }^{10}$ Aix Marseille

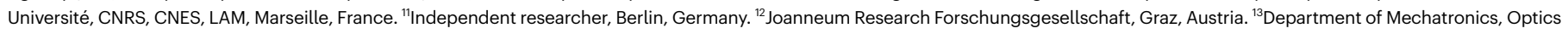
and Engineering Informatics, Budapest University of Technology and Economics, Budapest, Hungary. ${ }^{14}$ DLR Institute of Planetary Research, Berlin, Germany. ${ }^{15}$ Planetary Science Directorate, Southwest Research Institute (SwRI), Boulder, CO, USA. ${ }^{16}$ Max-Planck-Institut für Sonnensystemforschung, Göttingen, Germany. ${ }^{凶}$ e-mail: lorourke@esa.int 

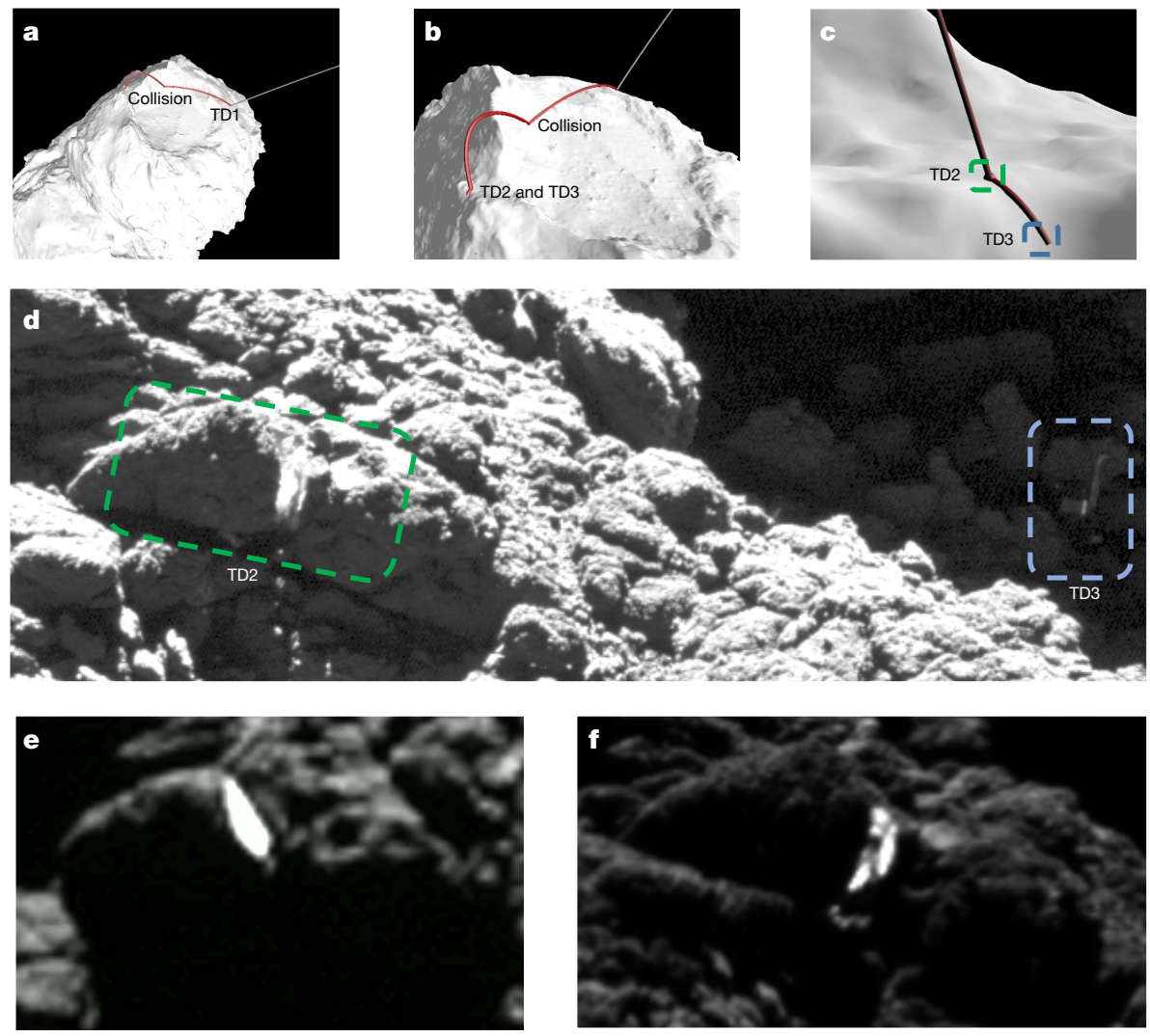

Fig. $1 \mid$ Philae landing trajectory, TD2 and TD3, Philae and visible ice. a-c, Three views showing the Philae landing trajectory as it crosses the surface of the comet (represented by a shape model) highlighting the locations of touchdown 1(TD1), collision, touchdown 2 (TD2) and touchdown 3 (TD3). d, OSIRIS image (2 September 2016, 19:59 UT; 0.049 m per pixel), showing locations of TD2 and TD3 as boxed in c. This image is enhanced in order to show the skull-top crevice (inside the green dashed box marked TD2) and the Philae lander hidden in the distant shadows (inside the blue dashed box marked TD3). e,f, Views of the ice in the crevice (6 August 2016 and 24 August 2016, respectively). location $^{11}$. A comparative analysis was performed of this area using pre- and post-landing imagery (Supplementary Figs. 2,4 ) from the Rosetta Optical, Spectroscopic, and Infrared Remote Imaging System $(\text { OSIRIS })^{14}$, combined with high-resolution digital terrain models of the ridge (for example, Supplementary Figs. 9-11) and of the Abydos region as a whole. While notable changes in boulder positions were observed in the Abydos valley (Supplementary Fig. 4e,f), the geomorphological structures along the length of the ridge showed no differences with regards to position or orientation. Changes were found however in the pre- and post-landing surface morphology of two adjoining boulders located on the ridge (Extended Data Fig. 1, Supplementary Video 1, Supplementary Figs. 5, 6). These changes included the identification of an unusual ice feature located in the boundary between the boulders (Fig. 1e,f, Supplementary Video 2). Our analysis of these changes found that only Philae's presence could explain their existence (Supplementary Methods). As the topological structures of these two boulders, viewed from above, give the impression of a skull face as shown in Supplementary Fig. 3, we chose to name the location 'skull-top ridge' and the boundary between the two boulders as 'skull-top crevice'. We highlight in Methods that the boulders themselves represent assemblages of dust/ice aggregates.

The timing involved in this chain of events (Extended Data Table 2, Supplementary Video 3) was derived from magnetometer data produced by the ROMAP sensor and checked against thermal and power information from Philae's subsystems. The ROMAP instrument provided attitude information (combined with the Rosetta Plasma Consortium Fluxgate Magnetometer, RPC-MAG ${ }^{15}$ ), as well as unique accelerometer measurements based on sensor boom movement (see Methods). Combined with our image analysis, a reassessment of the ROMAP science data (see Methods and Supplementary Methods) determined that the initial contact took place at 17:23:48 $\pm 10 \mathrm{~s}$ UT, approximately 1.5 min before the previously published contact time ${ }^{16}$. Indeed Philae spent nearly two full minutes at TD2, making four surface contacts in its trip across it (see Supplementary Fig. 6). Of the four, the third (TD2c) of these contacts (Fig. 2) is the most notable due to the 0.25 -m-deep depression visible in an ice-like feature on the side of the crevice. We found a perfect correlation between the presence of that depression and ROMAP boom movements (Fig. 2a) that shows the expected deviations matching the stamping movement required for its creation. The compression lasted $3 \mathrm{~s}$ (Fig. 2b, c) before Philae proceeded to rise out of the crevice to then make its final TD2 contact with the surface (TD2d), creating the 'eye' of the 'skull' in the process.

Data from the OSIRIS and VIRTIS (Visible, InfraRed and Thermal Imaging Spectrometer) instruments on the Rosetta orbiter were used to determine whether the high-albedo ice-like features observed in the skull-top crevice were water ice. For the OSIRIS instrument, we focused on multi-filter images generated during the timeframe of 12 to 14 June 2016 (Fig. 3a, Extended Data Fig. 2). A spectrophotometric analysis of the data sets from this period (see Methods) provided spatially resolved data that confirmed the presence of water ice in the crevice, matching a visible area of approximately $3.5 \mathrm{~m}^{2}$, with a brightness 6 times greater than that of the dust-covered terrain. The water-ice abundance was derived from the observed reflectance, after corrections were made for the illumination conditions and phase function based on geographical mixtures of the comet's dark terrain and water ice (grain size of $30-100 \mu \mathrm{m})$ applied to the bright material's absolute reflectance ${ }^{17}$. A water-ice abundance value of $46.4 \% \pm 2.0 \%$ was measured during the 14 June 2016 observations at 10:30:32 UT (Fig. 3c, d, Extended Data 


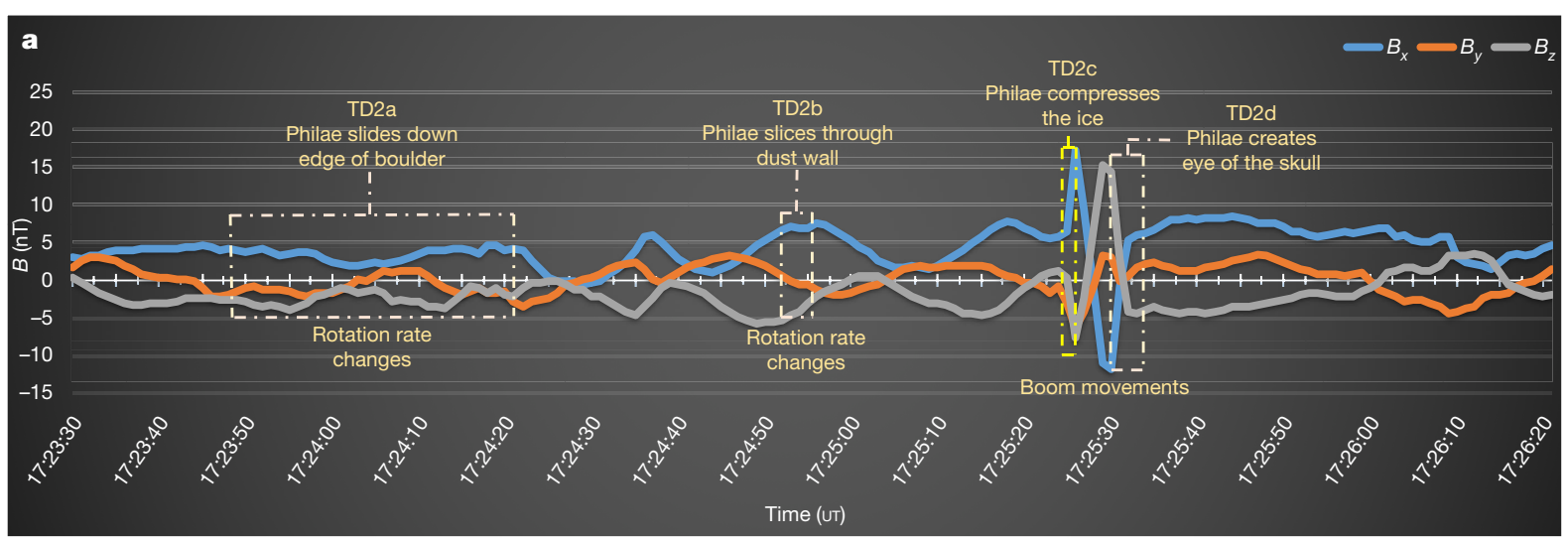

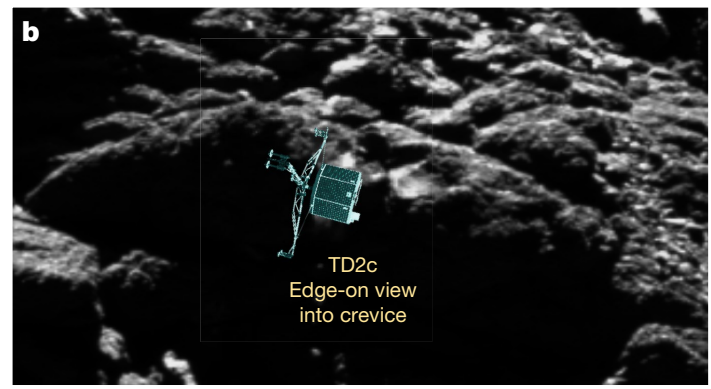

Fig. 2 | ROMAP guide and Philae impact TD2c. a, ROMAP magnetometer rotation and boom measurements matching Philae touchdown events $(\mathrm{TD} 2 \mathrm{a}-\mathrm{d}$ ) are plotted as magnetic field values versus time (see Methods and Supplementary Methods) whereby $B_{x}, B_{y}$ and $B_{z}$ represent the three magnetic field components. b, OSIRIS image focussing on TD2c, where the lander
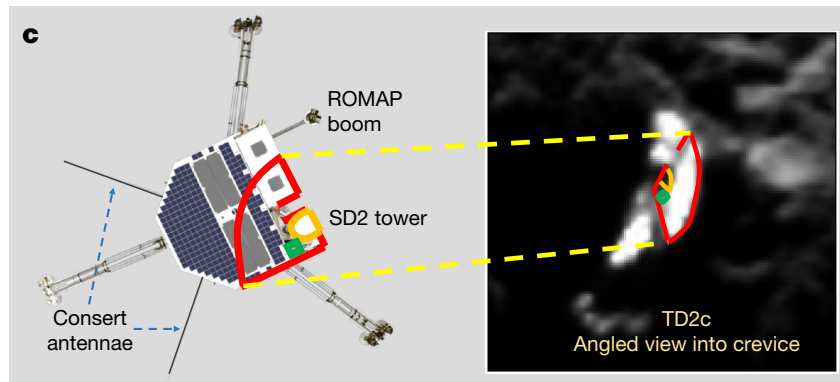

( $1 \mathrm{~m}$ width in the image) compressed the ice in the crevice (2September 2016). c, Left, overhead view of the Philae lander highlighting its instruments. The red, orange and green markings map to the impression in the ice (right; OSIRIS image from 24 August 2016). See Supplementary Video 2 showing a fly-over of the crevice and Supplementary Video 3 for an animation of this figure.
Table 1), resulting in an approximate local dust/ice volume ratio of $1.15_{-0.08}^{+0.1}: 1$. Assuming a dust/ice bulk density ratio of $\mathrm{two}^{18}$, and a similar porosity for dust and ice material, the local dust/ice mass ratio is approximately $2.3_{-0.16}^{+0.2}: 1$. This value is below the average dust/ice mass ratio for the nucleus, which is $>3$ for most estimates derived from measured data ${ }^{19}$.

Lower spatial resolution data from the VIRTIS Instrument on 14 June 2016 confirmed this detection of water ice. Figure 3 b shows the resulting hyperspectral image of the Abydos region with the skull-face crevice identified at the edge of the field of view. A signal from the ice located in the crevice was found in the tail of the optical point spread function (see Methods). An estimation by VIRTIS of the water-ice abundance in the location concurred with that of the OSIRIS measurement whereby the water-ice-rich spot was determined to have an approximate area of $1.27 \pm 0.5 \mathrm{~m}^{2}$ (matching the upper bound of $48 \%$ of $3.5 \mathrm{~m}^{2}$ ), calculated from the inferred abundances $\left(0.5 \%\right.$ over an area of $\left.253 \mathrm{~m}^{2}\right)$.

The general longevity of ice on the comet's surface is dependent on local surface topography ${ }^{6,17,20,21}$, with most ice features disappearing quite quickly owing to limited surface shading from the Sun within days to weeks of discovery. Local dust/ice ratios of $<3: 1$, equivalent to that in the skull-top crevice, have been found at other locations across the comet (as well as on other comets ${ }^{7,22}$ )-in particular, in newly exposed water ice observed on cliffs and scarps linked in some cases to outbursts as well as in clustered bright spots in both hemispheres ${ }^{4,6,17}$. As dust/ice ratios have been found to increase over time due to solar illumination exposure, the facts that our measurement of high water-ice abundance was made 19 months post-landing, and that the ice in the crevice was observed 22 months post-landing without notable measurable changes, both point to the ice in the crevice receiving very low solar illumination due to shadowing.

We confirmed this using a horizon mask (Extended Data Fig. 3), determining that the ice on the left-hand side of the crevice was illuminated
$<0.55 \%$ of the time during the perihelion passage while the ice on the right-hand side of the crevice, where the compression took place, received $<0.21 \%$ of direct sunlight during the same period. Using as input the corresponding energy flux, we found our sublimation modelling over-estimated by an order of magnitude the amount of ice that sublimed (compared to that visible in the imagery), pointing to even greater morphological shadowing than our horizon mask could derive (Supplementary Methods). This very low illumination is supported by our direct measurements of the crevice dimensions; the width of the TD2c location matches the width of the Philae lander, thus pointing to very little sublimation or erosion having taken place (see e1 in Fig. 3e). For these reasons, we conclude that while the super-volatiles may have sublimed over time, the water ice itself at TD2c did not sublime and remained in a highly unprocessed state.

The depth of the impression made by Philae in this icy surface, combined with a detailed correlation of the ROMAP boom measurements, contribute to direct in situ measurements allowing an estimate to be made of the compressive strength of this dust/ice feature. The depth of the impression in the ice is $0.246 \pm 0.049 \mathrm{~m}$ (e2 in Fig. 3e) and its area is $\geq 0.2208 \mathrm{~m}^{2}$ (see Methods). A detailed analysis was performed (Methods), and found the compressive strength of the ice to be $<12 \mathrm{~Pa}$. It is important to note that this very low compressive strength is of 'primitive' ice (see Supplementary Methods for explanation) buried and hidden from view until it was exposed and compressed at the time of the Philae landing itself.

Whereas compressive strengths $\mathrm{s}^{1,2}$ of $1 \mathrm{kPa}$ and $2 \mathrm{MPa}$ were measured at TD1 and TD3 respectively (although deployment uncertainties do affect the reliability of the MUPUS-Multi Purpose Sensors for Surface and Subsurface Science-penetrator result), a number of other publications find much lower values. Model-dependent analyses of the collapse of cliff overhangs observed from orbit ${ }^{23}$, as well as those derived from the scratches Philae made at the final landing location ${ }^{11}$, calculated 


\section{Article}
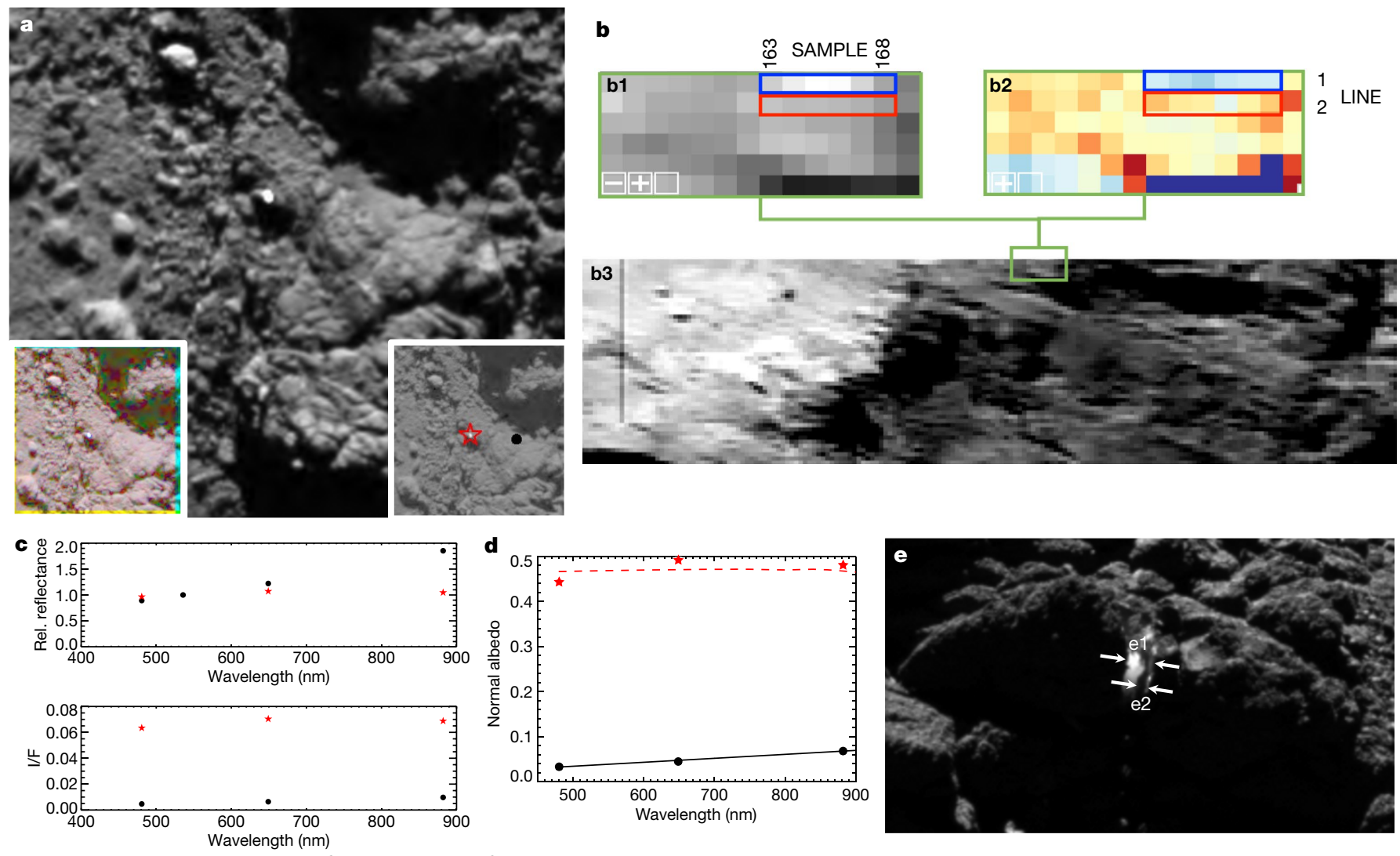

$\star$ Skull-top crevice $\bullet$ Comet dark terrain

Fig. 3 | Multi-instrument view of the water ice. a, Main panel, NAC image (taken on 14 June 2014 at 10:29 UT); insets, OSIRIS images (left, multi-filter view; right, star and black dot show positions for plots c, d). b, The $0.55 \mu \mathrm{m}$ VIRTIS-M hyperspectral cube (V1_00424522185.QUB) of the Abydos region (14 June 2016, 10:51-11:35 UT) with skull-top ridge located in the green box of the lower panel, b3. The upper panels b1 and b2 zoom-in on spectral slope (left) and the radiance factor (I/F) image (right), respectively. 'Sample' identifies the relevant pixel numbers in each 'Line' (row) of the cube. c, Plot of reflectance and I/F versus wavelength, and d, the measured albedo as a function of wavelength.e, OSIRIS image (2 September 2016) shows the crevice edge-on with e1 (crevice width) $1.03 \pm 0.07 \mathrm{~m}$ and $\mathrm{e} 2$ (compression width) $0.246 \pm 0.049 \mathrm{~m}$. compressive strength values ranging between $30 \mathrm{~Pa}$ and $150 \mathrm{~Pa}$ for the former and from $10 \mathrm{~Pa}$ to $100 \mathrm{~Pa}$ for the latter. Such model-based low compressive strength derivations show consistency with our in situ findings.

Three independent porosity estimations have been made for this comet, with values ranging from $65 \%-85 \%$ (Philae Consert radar $^{3,24}$ ) to $70 \%-75 \%$ (Rosetta Radio Science Instrument) ${ }^{25}$, and a modelled third estimate ${ }^{26}$ of $63 \%-79 \%$. These same numbers equate to volume filling factors of $0.15-0.35,0.25-0.3$ and $0.21-0.37$, respectively.

We modelled the compression Philae made in the cometary boulder material, with the aim of determining how much the volume-filling factor of the material is altered. The model we use $\mathrm{e}^{27}$ assumes that the material making up the cometary interior is made up of a hierarchical arrangement of building blocks (see Supplementary Methods). According to this model, applied here to the interior of a cometary boulder, the submicrometre-sized solid grains ${ }^{28,29}$ are contained in larger units ('pebbles'), which themselves are clustered together to make up the boulder.

We find that such an arrangement of hierarchical building blocks, which previously has demonstrated excellent correlation with the Philae Consert radar results ${ }^{24}$, also provides the conditions needed to achieve the low compressive strength $(<12 \mathrm{~Pa})$ of the material of the boulder into which Philae stamped (see Methods). We further note that although the local dust/ice ratio we measured $\left(2.3_{-0.16}^{+0.2}\right)$ is lower than the model-based average ${ }^{30}$ estimate of the nucleus (between 3 and 9), we can resolve this inconsistency. A study of results from gas activity models ${ }^{31,32}$ (based also on our hierarchical pebble model) explains our finding in that dust/ice ratios of 5 and lower can be found to be present locally for up to $5 \%$ of the volume of the nucleus ${ }^{29}$. As a result and on the basis of the consistencies found using our model, we derive a volume filling factor for the boulder material of $0.25 \pm 0.07$ (a porosity range of $68 \%-82 \%$ ), equivalent to previously published values for the overall nucleus of comet 67P.

Our results provide important constraints for future cometary lander missions, as the knowledge of cometary boulder interiors is not only vital for impact analysis but also provides insights into the mechanical processes needed to retrieve a volatile-rich cryogenic sample for in situ analysis-or indeed for delivery back to the Earth ${ }^{33,34}$. The operational dangers of landing in a cometary boulder field would however need careful study and preparation.

\section{Online content}

Any methods, additional references, Nature Research reporting summaries, source data, extended data, supplementary information, acknowledgements, peer review information; details of author contributions and competing interests; and statements of data and code availability are available at https://doi.org/10.1038/s41586-020-2834-3.

1. Biele, J. et al. The landing(s) of Philae and inferences about comet surface mechanical properties. Science 349, aaa9816 (2015).

2. Spohn, T. et al. Thermal and mechanical properties of the near-surface layers of comet 67P/Churyumov-Gerasimenko. Science 349, aab0464 (2015).

3. Kofman, W. et al. Properties of the 67P/Churyumov-Gerasimenko interior revealed by CONSERT radar. Science 349, aab0639 (2015). 
4. Pajola, M. et al. The pristine interior of comet $67 \mathrm{P}$ revealed by the combined Aswan outburst and cliff collapse. Nat. Astron. 1, 0092 (2017).

5. Fornasier, S. et al. Surface evolution of the Anhur region on comet 67P/ Churyumov-Gerasimenko from high-resolution OSIRIS images. Astron. Astrophys. 630 A13 (2019).

6. Oklay, N. et al. Long-term survival of surface water ice on comet 67P. Mon. Not. R. Astron. Soc. 469, S582-S597 (2017).

7. Oklay, N. et al. Comparative study of water ice exposures on cometary nuclei using multispectral imaging data. Mon. Not. R. Astron. Soc. 462, S394-S414 (2016).

8. Luccheti, A. et al. Characterization of the Abydos region through OSIRIS high-resolution images in support of CIVA measurements. Astron. Astrophys. 585, L1 (2016).

9. Keller, H. U. et al. Seasonal mass transfer on the nucleus of comet $67 \mathrm{P} / \mathrm{Churyumov}$ Gerasimenko. Mon. Not. R. Astron. Soc. 469 (Issue Suppl. 2), S357-S371 (2017).

10. Glassmeier, K. et al. The Rosetta mission: flying towards the origin of the Solar System. Space Sci. Rev. 128, 1-21 (2007).

11. Heinisch, P. et al. Compressive strength of comet 67P/Churyumov-Gerasimenko derived from Philae surface contacts. Astron. Astrophys. 630, A2 (2019).

12. Heinisch, P. et al. Reconstruction of the flight and attitude of Rosetta's lander Philae. Acta Astronaut. 140, 509-516 (2017).

13. Auster, H. U. et al. ROMAP: Rosetta magnetometer and plasma monitor. Space Sci. Rev. 128, 221-240 (2007).

14. Keller, H. U. et al. OSIRIS - the scientific camera system onboard Rosetta. Space Sci. Rev. 128, 433-506 (2007).

15. Glassmeier, K. et al. RPC-MAG, the fluxgate magnetometer in the ROSETTA plasma consortium. Space Sci. Rev. 128, 649-670 (2007).

16. Auster, H. U. et al. The non-magnetic nucleus of comet 67P/Churyumov-Gerasimenko. Science 349, aaa5102 (2015).

17. Fornasier, S. et al. Rosetta's comet 67P/Churyumov-Gerasimenko sheds its dusty mantle to reveal its icy nature. Science 354, 1566-1570 (2016).

18. Fulle, M. et al. The dust-to-ices ratio in comets and Kuiper belt objects. Mon. Not. $R$. Astron. Soc. 469, S45-S49 (2017).

19. Choukroun, M. et al. Dust-to-gas and refractory-to-ice mass ratios of comet 67P/ Churyumov-Gerasimenko from Rosetta observations. Space Sci. Rev. 216, 44 (2020).

20. Deshapriya, J. D. P. Exposed bright features on the comet 67P/Churyumov-Gerasimenko: distribution and evolution. Astron. Astrophys. 613, A36 (2018).

21. Filacchione, G. et al. Exposed water ice on the nucleus of comet 67P/ Churyumov-Gerasimenko. Nature 529, 368-372 (2016).
22. Sunshine, J. M. et al. Exposed water ice deposits on the surface of comet 9P/Tempel 1 . Science 311, 1453-1455 (2006).

23. Groussin, O. et al. Gravitational slopes, geomorphology, and material strengths of the nucleus of comet 67P/Churyumov-Gerasimenko from OSIRIS observations. Astron. Astrophys. 583, A32 (2015).

24. Herique, A. et al. Homogeneity of 67P/Churyumov-Gerasimenko as seen by CONSERT: implication on composition and formation. Astron. Astrophys. 630, A6 (2019).

25. Pätzold, M. et al. A homogeneous nucleus for comet 67P/Churyumov-Gerasimenko from its gravity field. Nature 530, 63-65 (2016).

26. Fulle, M. et al. Comet $67 \mathrm{P} /$ Churyumov-Gerasimenko preserved the pebbles that formed planetesimals. Mon. Not. R. Astron. Soc. 462, S132-S137 (2016).

27. Blum, J. et al. The physics of protoplanetesimal dust agglomerates. I. Mechanical properties and relations to primitive bodies in the Solar System. Astrophys. J. 652, 17681781 (2006).

28. Mannel, T. et al. Dust of comet 67P/Churyumov-Gerasimenko collected by Rosetta/ MIDAS: classification and extension to the nanometre scale. Astron. Astrophys. 630, A26 (2019).

29. Güttler, C. et al. Synthesis of the morphological description of cometary dust at comet 67P/Churyumov-Gerasimenko. Astron. Astrophys. 630, A24 (2019).

30. Lorek, S., Gundlach, B., Lacerda, P. \& Blum, J. Comet formation in collapsing pebble clouds - what cometary bulk density implies for the cloud mass and dust-to-ice ratio. Astron. Astrophys. 587, A128 (2016).

31. Fulle, M. et al. How comets work: nucleus erosion versus dehydration. Mon. Not. $R$. Astron. Soc. 493, 4039-4044 (2020).

32. Gundlach, B., Fulle, M. \& Blum, J. On the activity of comets: understanding the gas and dust emission from comet 67P/Churyumov-Gerasimenko's south-pole region during perihelion. Mon. Not. R. Astron. Soc. 493, 3690-3715 (2020).

33. Bockelée-Morvan, D. et al. AMBITION-Comet Nucleus Cryogenic Sample Return. https://www.cosmos.esa.int/web/voyage-2050/white-papers (ESA, 2019)

34. Veverka, J. Cryogenic Comet Nucleus Sample Return (CNSR) Mission Technology Study. Report SDO-12367 https://solarsystem.nasa.gov/studies/228/cryogenic-comet-nucleussample-return-cnsr-mission-technology-study (NASA, 2017).

Publisher's note Springer Nature remains neutral with regard to jurisdictional claims in published maps and institutional affiliations.

(c) The Author(s), under exclusive licence to Springer Nature Limited 2020 


\section{Methods}

\section{OSIRIS data analysis}

The normal albedo presented here has been evaluated from photometrically corrected images using the shape 7S model with 12 million facets ${ }^{35}$ and the Hapke model ${ }^{36}$ parameters (table 4 of ref. ${ }^{37}$ ) from resolved photometry in the orange filter centred at $649 \mathrm{~nm}$.

We assume that the phase function at $649 \mathrm{~nm}$ also applies at the other wavelengths ${ }^{17}$. The flux of a region of interest (ROI) in each of the 3 filters has been integrated over 3-pixel-wide, square boxes. We attempt to reproduce the spectral behaviour and the normal albedo of the ice-rich patches by obtaining synthetic spectra of areal mixtures (spatially segregated) of the comet's dark terrain (DT), derived from areas near the boulder with water ice:

$$
R=\rho R_{\text {ice }}+(1-\rho) R_{\mathrm{DT}}
$$

where $R$ is the reflectance of the bright patches, $R_{\text {ice }}$ and $R_{\mathrm{DT}}$ are the reflectance of the water ice and of the comet's dark terrain, respectively, and $\rho$ is the relative surface fraction of water ice or frost.

We use areal mixture models due to the absence of reliable and relevant optical constants for the dark material needed to run more complex scattering models, and the absence of clear absorption features in the wavelength range covered by the OSIRIS observations. The water-ice spectrum was derived from Hapke modelling of optical constants ${ }^{38}$ using grain sizes of $30 \mu \mathrm{m}$ and $100 \mu \mathrm{m}$. In fact, the typical size of ice grains on cometary nuclei was found to be a few tens of micrometres $22,39,40$.

We also attempt to use models with larger water-ice grains (up to $1,000 \mu \mathrm{m}$ and $2,000 \mu \mathrm{m}$ ), but these models gave a worse spectral match and a lower $\chi^{2}$ fit. The models that best fit the maximum absolute reflectance observed on the bright patch on the skull boulder are areal mixtures of the average cometary dark terrain (DT) enriched with $46.4 \%-47.4 \%$ of water ice (Fig. $3 d$, Extended Data Table 1 ) with grain sizes of $30-100 \mu \mathrm{m}$. A further analysis is provided in Supplementary Methods.

\section{VIRTIS data analysis}

The best viewing opportunity for VIRTIS- $\mathrm{M}^{41}$ of the Philae touchdown 2(TD2) site occurred on 14 June 2016 between 10:51:12 and 11:35:31 UTC when the Rosetta spacecraft was at a distance of $27.3 \mathrm{~km}$ from $67 \mathrm{P}$ and the solar phase angle was $57^{\circ}$.

During this period of time, the VIRTIS-M instrument acquired a visible hyperspectral cube (acquisition V1_00424522185.QUB) by collecting 133 consecutive slit images of the surface with a spatial resolution of about $6.5 \times 10 \mathrm{~m}$ (along slit $\times$ scan). Each slit image was acquired with an integration time of $16 \mathrm{~s}$ and a repetition step time of $20 \mathrm{~s}$ while the Rosetta spacecraft was maintaining nadir pointing. At each step the internal scan mirror is rotated by an angle of $250 \mu \mathrm{rad}$ (corresponding to one Instantaneous Field-Of-View-IFOV) to achieve an angular scan of about $1.9^{\circ}$ in 133 steps. Consecutive slits are not completely connected among them, being separated by about $13 \mathrm{~m}$.

The resulting hyperspectral image of the Abydos region shown in Fig. $3 \mathrm{~b}$ has a scale of $1.66 \mathrm{~km}$ (slit width) by $0.86 \mathrm{~km}$ (scan length). The position of the cracked boulder on the TD2 site has been identified close to pixels at line $=1$, samples $=163-168$ and appears located at the edge of the FOV (see Fig. 3b, panel b2, blue box). According to reconstructed geometries computed on the SHAP7 digital model and including the current best estimate of the errors, the centres of these pixels are offset by a minimum of $25 \mathrm{~m}$ to a maximum of $58 \mathrm{~m}$ with respect to the reference position of TD2. The identification of the TD2 location on the VIRTIS image is therefore not fully certain owing to the limited spatial resolution and position of the pixels on the edge of the VIRTIS image.

Owing to the coarse spatial resolution, VIRTIS-M is not able to resolve the cracked boulder whose exposed bright area is about $0.5 \mathrm{~m}^{2}$ while the pixel area is $65 \mathrm{~m}^{2}$. Moreover, owing to the instrumental point spread function ${ }^{42,43} \mathrm{FWHM}(<500 \mu \mathrm{rad})$ and to the uncertainty on the position of the TD2 site, we are averaging the signal of the candidate TD2 area taken on 6 contiguous pixels where higher reflectance and blueing is observed.

The average reflectance spectrum of the TD2 pixels is shown in Extended Data Fig. 4a and compared with an average collected on nearby pixels (line $=2$, samples $=163-168$ ) as a reference for an adjacent non-icy terrain (Extended Data Fig. 4b). The analysis of the spectral slope measured on the two ROI gives a value of $2.39 \mu^{-1}$ and $2.59 \mu^{-1}$ for the cracked boulder pixels (blue curve) and nearby dark terrain (red curve), respectively. These values correspond to a spectral slope difference of $\Delta=0.20 \mu^{-1}$. This difference is equivalent ${ }^{44}$ to a water-ice abundance of $0.1 \%$ in areal mixing (Extended Data Fig. $4 \mathrm{c}$ ).

The size of the bright area on TD2 has been constrained to about $3.5 \mathrm{~m}^{2}$ on OSIRIS high-resolution images, of which about $1 \mathrm{~m}^{2}$ is made of exposed water ice in an areal mixture (where water ice and dust do not thermally interact leading to less energy being available for sublimation). Our analysis shows that VIRTIS is missing the TD2 location by about one line and that the signal is harvesting the tail of the optical point spread function. As a consequence of this, we are collecting about $20 \%$ of the photons coming from the water-ice patch on the TD2 site. This means that the water-ice abundance of $0.1 \% \pm 0.04 \%$ previously estimated is likely to be five times larger, leading to $0.5 \% \pm 0.2 \%$. Scaling this value for the total area of $253 \mathrm{~m}^{2}$ on 6 pixels, the water-ice-rich spot corresponds to an area of about $1.27 \pm 0.5 \mathrm{~m}^{2}$, in agreement with OSIRIS findings.

\section{ROMAP and RPC-MAG data analysis}

The tri-axial lander magnetometer ROMAP and orbiter magnetometer RPC-MAG were operating during the descent, landing and rebound phase with a sampling rate of $1 \mathrm{~Hz}$. Extended Data Fig. 5 shows the measurements of both instruments for the interval around TD2. In order to use magnetic field measurements for flight dynamics reconstructions, reference measurements are necessary to separate external events in the background magnetic field (for example, magnetic wave activity as seen in the RPC-MAG observations in Extended Data Fig. 5) from changes caused by the spacecraft dynamics or operation (for example, rotation change or boom movements). In this case, the RPC-MAG orbiter instrument was used as background field reference to be able to reconstruct the Philae dynamics.

A rotation of the lander along an arbitrary axis relative to the background magnetic field causes an apparent rotation of the magnetic field vector observed by the lander relative to the orbiter reference. This causes the three-dimensional (3D) quasi-sinusoidal modulation of the ROMAP measurements relative to the RPC-MAG measurements visible in Extended Data Fig. 5. A time-dependent rotation matrix between these two 3D observations can therefore be calculated to describe the attitude of the lander relative to the orbiter. This information can then in theory be used to derive a set of time-dependent quaternions giving the absolute attitude of the lander.

A statistical analysis has to be used to accurately determine the absolute attitude to account for small deviations between the orbiter and lander measurements caused by noise and plasma phenomena (for a detailed description, see ref. ${ }^{12}$ ). In this case only a minimal number of data points is available due to the low $1 \mathrm{~Hz}$ sampling rate and relatively fast changes in lander dynamics. Hence, a simplified analysis for the reconstruction of the rotation rate during descent was used ${ }^{12}$ to estimate the average lander rotation rate between the individual surface contacts (see Extended Data Table 2). Instead of determining the total absolute lander orientation, this method is based on determining only the orientation of the lander rotation axis, which allows the lander magnetic field observations to be transformed into a temporary coordinate system in which one magnetic axis remains stable (that is, the field along the rotation axis) and only the two remaining axes show a 
modulation. This modulation relative to the orbiter reference measurements can easily be determined, and results directly in the lander rotation frequency ${ }^{12}$.

A rotation of the magnetometer boom relative to the lander creates a characteristic signature (as can be seen around 17:25:30 UT in Fig. 2a and Extended Data Fig. 5) in the magnetic field caused by the displacement of the ROMAP sensor relative to the static lander bias field ${ }^{13}$. The shape and duration of this signature allows us to constrain the acceleration acting on the lander perpendicular to the boom rotation axis, that is, along the lander $z$ axis. The other touchdowns ${ }^{1,11}$ were used as references to determine the direction and timing, and gain a qualitative insight into the magnitude (see Supplementary Information for more details)

\section{Philae lander dynamics at the TD2c point}

This section covers only the dynamics at the TD2c point, where the ice was compressed by the Philae balcony and SD2 tower. The full dynamics that took place through all four TD2 points (TD2a-d) are described in the Supplementary Methods and summarized in Extended Data Table 2.

The TD2c contact duration is linked to the ROMAP boom, which showed an upwards movement at 17:25:24 \pm 1 s continuing until approximately 17:25:27 \pm 1 s (Fig. 2a, Extended Data Fig. 5). At 17:25:27 $\pm 1 \mathrm{~s}$, the boom started to move downwards away from the lid, meaning that the acceleration of the lander had stopped or reversed. The change at 17:25:27 $\pm 1 \mathrm{~s}$ is considered the end position of the stamping in TD2c, because the geometry from the deceleration of Philae during stamping causes an upward deflection of the boom, which is what was observed. This resulted in a duration of $3 \pm 1 \mathrm{~s}$ for the stamping/compression of the ice (see Fig. 2a-c and the animation in Supplementary Video 3). The energy loss at TD2c caused by the stamping is estimated to be $0.671 \pm 0.297 \mathrm{~J}$.

\section{Estimating TD2c surface area and depth}

The images used in the data analysis are obtained from numerous epochs and distances from the comet. The pixel resolution linked to distance ranged from $0.16 \mathrm{~m}$ per pixel in May 2016 when the spacecraft was $8.5 \mathrm{~km}$ from the crevice, to $0.13 \mathrm{~m}$ per pixel at a distance of $7 \mathrm{~km}$ in June 2016, to $0.049 \mathrm{~m}$ per pixel at the closest distance reached on 2 September 2016 (2.63 km). The pixel size in Extended Data Fig. 1 for both pre-and post-landing images was $0.15 \mathrm{~m}$ per pixel, as the images were taken at an equivalent distance $(8 \mathrm{~km})$ to the crevice.

Image analysis relied primarily on cross-correlating multiple images to determine accurate estimates of heights and width of different boulder features. The lower the resolution the greater the error bar, as it became difficult to determine where the edge of a feature actually started or ended owing to the greater coverage of the pixel. The error bar is therefore linked to the pixel resolution achievable in the image analysis.

A lower limit of $0.2208 \mathrm{~m}^{2}$ has been estimated for the area of ice compressed by Philae in the TD2c position. Only two OSIRIS images ( 21 August 19:19 UT, 24 August 2016 19:39 UT; Figs. 1f, 2c) provide a clear, albeit angled, view of the ice in the compression. The full width of the ice impression could therefore not be estimated due to lack of direct visibility. It was feasible however to use both these images as well as another from 2 September 2016 19:59 in Fig. 3e (which provides an edge-on-view of the ice impression) to obtain a lower-limit measurement of the length of the sides of the Philae balcony that made the impression. The estimate is a combination therefore of the actual area of the Philae balcony matching these lengths plus the area of an arc matching the remainder of the angled visible ice.

Figures 1d, 2b and 3e (same images) were taken on 2 September 2016 at the closest distance $(2.63 \mathrm{~km})$ to the nucleus surface achieved by Rosetta during the mission, and provide therefore the highest resolution. This image, which was the famous Philae discovery image, provides a clear high-resolution view of the edge of the compressed ice region in TD2c. The solar illumination in this image is equivalent to the illumination observed on 6 August 05:52 UT, and allows to conclude that while the sunlight was gradually moving down the crevice, it had not yet arrived at the compressed region. As a result, the stamped edge lies in shadow, with the exposed ice further back in the crevice creating a back-light effect. This edge-on view allows an accurate measurement of $0.246 \pm 0.049 \mathrm{~m}$ to be taken of the depth of the compressed region. In that respect, the one image showing Philae resting on the surface of the comet (Fig. 1d) also provides the key input measurement for this paper. Further dimension-related images are provided (Supplementary Figs. 7, 8).

\section{Compressive strength and porosity analysis}

The model that we use. To derive material properties for the cometary boulder from our findings, we consider a hierarchical setup of the interior structure of the boulder assuming the dust and ice grains are concentrated in larger units ('pebbles'), which themselves are clustered together to make up the boulder ${ }^{45}$. Thus, the boulder itself is a kind of 'rubble pile' and possesses pore space on two length scales, the microscopic and the pebble-sized. Comparisons between our model and others addressing the basic building blocks of the internal material of the comet can be found elsewhere ${ }^{24,32,46,47}$ and are not dealt with further in this paper.

Compressivestrength estimates. As the incoming trajectory of Philae at TD2c with respect to the surface normal shows, Philae lost a total kinetic energy of $\Delta E=0.671 \pm 0.297$ J owing to compression ('stamping') of the surface material of comet 67P. The vertical component of the incoming motion of Philae resulted in the compaction of the porous cometary matter. The characteristic stress required for stamping is determined by the compressive strength $P_{\mathrm{C}}$ of the material. With the depth and minimum area of the stamping impression Philae made estimated (see main text) to be $h=0.246 \pm 0.049 \mathrm{~m}$ and $A_{\mathrm{Min}}=0.2208 \mathrm{~m}^{2}$, respectively, a minimum volume of $V_{\mathrm{Min}}=A_{\mathrm{Min}} h=0.054 \mathrm{~m}^{3}$ was displaced during stamping.

We can make a first, crude, estimate of the compressive strength by assuming that the compressed volume is at least $V_{\text {Min }}$ and that the compressive strength is a constant material value, that is, is independent of the compressional state of the matter. In reality, a much larger volume than $V_{\mathrm{Min}}$ is affected by the impact of Philae and $P_{\mathrm{C}}$ is a strong function of the porosity (see below). However, we can state that $P_{\mathrm{C}}<\frac{\Delta E}{V_{\text {Min }}}=12 \mathrm{~Pa}$ is required to account for the observed material compression and energy loss of Philae. It should be noted that this upper limit for the compressive strength of 67P's surface material is model independent and based on firm measurements (this paper).

Compressive strength link to volume filling factor. The compressive strength is not a constant material value, but rather depends on the volume filling factor (fraction of total volume filled by material), $\Phi$, in a characteristic way $27,48,49$

$$
P_{\mathrm{C}}(\Phi)=p_{\mathrm{m}}\left(\frac{\Phi-\Phi_{1}}{\Phi_{2}-\Phi}\right)^{\Delta^{\prime}}
$$

with $\Phi_{1}$ and $\Phi_{2}$ being the formal lower and upper limits of the volume filling factor for which $P_{\mathrm{C}}\left(\Phi_{1}\right)=0$ and $P_{\mathrm{C}}\left(\Phi_{2}\right) \rightarrow \infty$, respectively (see below for $p_{\mathrm{m}}$ and $\left.\Delta^{\prime}\right)$.

Experiments and numerical simulations showed ${ }^{27,48-50}$ that $\Phi_{2}$ is in the range $0.1-0.9$, depending on the particle properties, such as grain size, grain-size distribution, grain shape, and the mode of deposition or compression. The value of $\Phi_{1}$, which has no physical meaning and is merely a fitting parameter, ranges ${ }^{45}$ between 0.05 and 0.35 . The factor $p_{\mathrm{m}}$ is the characteristic compressive strength and $\Delta^{\prime}$ describes the logarithmic range of stresses in which compression takes place: that is, most compression happens in the interval $\left(10^{-\Delta^{\prime}} p_{\mathrm{m}}, 10^{+\Delta^{\prime}} p_{\mathrm{m}}\right)$.

Schräpler et al. ${ }^{49}$ showed that this relation holds for a wide range of particle sizes, from loose granular ensembles of dust aggregates (the 'pebbles' in our notation) to others of a more homogeneous 
assemblage. They also showed that $\Delta^{\prime}=1.3$ is an appropriate value for all kinds of grain and pebble sizes. Pebble assemblages possess characteristic compressive strengths of $p_{\mathrm{m}} \approx 6.1 \times 10^{-2} \mathrm{~Pa}$ for pebbles with $1 \mathrm{~mm}$ radius and $p_{\mathrm{m}} \approx 4.7 \times 10^{-3} \mathrm{~Pa}$ for pebbles with $1 \mathrm{~cm}$ radius (for the heterogeneous case-as is our model), whereas submicrometre-sized particles in a more homogenous structure are compressed with $p_{\mathrm{m}} \approx 10^{4} \mathrm{~Pa}$. The schematic functional dependence of the volume filling factor on compression for our model is shown in Extended Data Fig. 6c.

Volume filling factor applied to the cometary boulderinterior (TD2c). To estimate the volume filling factor, and therefore the porosity, we need to take into account the manner in which the pebble assemblage compacts when the boulder interior is formed. The different ways in which this can happen can vary, as touched on briefly in the Supplementary Information, whether it occurs at initial cometary formation or long afterwards as the result of cometary dynamical events. The resulting packing fraction of the pebble assemblage can be defined in these scenarios by random loose packing (RLP), which means ${ }^{51} \Phi_{\mathrm{RLP}} \approx 0.55$, if inter-pebble friction is strong ${ }^{52}$ (a criterion satisfied for dust/ice aggregates) and if the size-frequency distribution of the pebbles is narrow (see Supplementary Methods).

The maximum random packing density is random close packing (RCP), or $^{51,52} \Phi_{\mathrm{RCP}} \approx 0.64$ for narrow size distributions. Thus, Philae's energy was dissipated by the compaction from RLP towards RCP (see Extended Data Fig. 6c). The total compressed volume must be larger than the displaced volume, $V_{\mathrm{Min}}$, to make room for the displaced material (Extended Data Fig. 6a, b). Assuming that the overall compressed volume is $V_{\mathrm{Eff}}=\eta V_{\mathrm{Min}}$ and that the increase in volume filling factor from initially $\Phi_{\mathrm{Min}}=\Phi_{\mathrm{RLP}}$ to $\Phi_{\mathrm{Max}}$ is identical everywhere inside this volume, the volume scaling factor can be derived as $\eta=\frac{\Phi_{\mathrm{Min}}}{\delta \phi}$, with $\delta \Phi=\Phi_{\mathrm{Max}}-\Phi_{\mathrm{Min}}$. We can calculate the energy dissipated by Philae's stamping motion (in the $z$ direction), $\Delta E=0.671 \mathrm{~J}$ to be:

$$
\begin{aligned}
& \Delta E=\int_{V_{\mathrm{Eff}}+V_{\mathrm{Min}}}^{V_{\mathrm{Eff}}} P_{\mathrm{C}} \mathrm{d} V \\
& =A \int_{0}^{h} P_{\mathrm{C}} \mathrm{d} z \\
& =A p_{\mathrm{m}} \int_{0}^{h}\left(\frac{\Phi(\mathrm{z})-\Phi_{1}}{\Phi_{\mathrm{RCP}}-\Phi(\mathrm{z})}\right)^{\Delta^{\prime}} \mathrm{d} z \\
& =A p_{\mathrm{m}} \int_{\Phi_{\text {Min }}}^{\Phi_{\mathrm{Max}}}\left(\frac{\Phi-\Phi_{1}}{\Phi_{\mathrm{RCP}}-\Phi}\right)^{\Delta^{\prime}} \frac{\mathrm{dz}}{\mathrm{d} \Phi} \mathrm{d} \Phi \\
& =\frac{V_{\mathrm{Min}} p_{m}}{\delta \Phi} \int_{\Phi_{\mathrm{Min}}}^{\Phi_{\text {Max }}}\left(\frac{\Phi-\Phi_{1}}{\Phi_{\mathrm{RCP}}-\Phi}\right)^{\Delta^{\prime}} \mathrm{d} \Phi,
\end{aligned}
$$

in which we use equation (2) with $\Phi_{2}=\Phi_{\mathrm{RCP}}$ and the approximation $\Phi(z)=\Phi_{\text {Min }}+\frac{z}{h} \delta \Phi$ and $\frac{\mathrm{d} z}{\mathrm{~d} \phi}=\frac{h}{\delta \Phi}$, with $\mathrm{h}=0.246 \mathrm{~m}$ and $A=0.2208 \mathrm{~m}^{2}$ being Philae's intrusion and stamping cross-section.

Applying equation (3) (see Supplementary Table1) with the nominal values $p_{\mathrm{m}} \approx 10^{-2} \mathrm{~Pa}, \Phi_{\mathrm{Min}}=\Phi_{\mathrm{RLP}}=0.55, \Delta^{\prime}=1.3$ and $\Phi_{1}=0.05$ shows that $\Phi_{\mathrm{Max}} \approx \Phi_{\mathrm{RCP}}$ and, thus, $\eta=6.1$ and $V_{\mathrm{Eff}}=0.33 \mathrm{~m}^{3}$.

It should be noted that the volume filling factor assumed in the above calculation, $\Phi=\Phi_{\mathrm{RLP}} \approx 0.55$, is that of the pebble assemblage. The pebbles themselves consist of submicrometre-sized dust/ice particles so that they possess internal porosity, which can be expressed by their inner volume filling factor $\Phi_{\text {pebble }} \approx 0.33-0.58$, depending on the type of compression experienced by the pebbles, as shown in laboratory experiments $^{48}$.

Taking the average of this range in volume filling factor of the pebbles, $\Phi_{\text {pebble }}=0.455 \pm 0.125$, we get an overall volume filling factor of the boulder of $\Phi_{\text {boulder }}=\Phi_{\text {RLP }} \Phi_{\text {pebble }}=0.25 \pm 0.07$, comparable with the values determined by Rosetta ${ }^{24-26}$. It should be noted that our two-step hierarchical model for the inner constitution of the boulder is plausible, but by no means the only feasible solution (see Supplementary Methods for more details).

\section{Data availability}

All OSIRIS, VIRTIS, RPC-MAG and ROMAP calibrated data are publicly available through the European Space Agency's Planetary Science Archive website (https://archives.esac.esa.int/psa/). The Supplementary Information contains additional supporting images, data and explanatory text with the aim of allowing readers to understand what we have done and how we have done it.

35. Jorda, L. et al. The global shape, density and rotation of 67P/Churyumov-Gerasimenko from pre-perihelion Rosetta/OSIRIS observations. Icarus 277, 257-278 (2016).

36. Hapke, B. et al. Bidirectional reflectance spectroscopy. 5. The coherent backscatter opposition effect and anisotropic scattering. Icarus 157, 523-534 (2002).

37. Fornasier, S. et al. Spectrophotometric properties of the nucleus of comet 67P/ Churyumov-Gerasimenko from the OSIRIS instrument onboard the ROSETTA spacecraft. Astron. Astrophys. 583, A30 (2015).

38. Warren, S. G. \& Brandt, R. E. Optical constants of ice from the ultraviolet to the microwave: a revised compilation. J. Geophys. Res. 113, D14220 (2008).

39. Capaccioni, F. et al. The organic-rich surface of comet $67 \mathrm{P} /$ Churyumov-Gerasimenko as seen by VIRTIS/Rosetta. Science 347, aaa0628 (2015).

40. Filacchione, G. et al. The global surface composition of 67P/CG nucleus by Rosetta/ VIRTIS. (1) Prelanding mission phase. Icarus 274, 334-349 (2016).

41. Coradini, A. et al. VIRTIS: an imaging spectrometer for the Rosetta mission. Space Sci. Rev. 128, 529-559 (2007).

42. Filacchione, G. On-ground characterization of Rosetta/VIRTIS-M. II. Spatial and radiometric calibrations. Rev. Sci. Instrum. 77, 103106 (2006).

43. Ammannito, E. et al. On-ground characterization of Rosetta/VIRTIS-M. I. Spectral and geometrical calibrations. Rev. Sci. Instrum. 77, 093109 (2006).

44. Raponi, A. et al. The temporal evolution of exposed water ice-rich areas on the surface of 67P/Churyumov-Gerasimenko: spectral analysis. Mon. Not. R. Astron. Soc. 462 (Issue Suppl. 1), S476-S490 (2016).

45. Blum, J. et al. Evidence for the formation of comet 67P/Churyumov-Gerasimenko through gravitational collapse of a bound clump of pebbles. Mon. Not. R. Astron. Soc. 469, S755S773 (2017).

46. Skorov, Y. V. \& Blum, J. Dust release and tensile strength of the non-volatile layer of cometary nuclei. Icarus 221, 1-11 (2012).

47. Davidsson, B. J. R. et al. The primordial nucleus of comet 67P/Churyumov-Gerasimenko. Astron. Astrophys. 592, A63 (2016).

48. Güttler, C. et al. The physics of protoplanetesimal dust agglomerates. IV. Toward a dynamical collision model. Astrophys. J. 701, 130-141 (2009).

49. Schräpler, R. et al. The stratification of regolith on celestial objects. Icarus $\mathbf{2 5 7}$, 33-46 (2015).

50. Oquendo-Patiño, W. F. \& Estrada-Mejia, N. Optimal packing of poly-disperse spheres in 3D: effect of the grain size span and shape. In Proc. VI International Conference on Particle-based Methods-Fundamentals and Applications (eds Oñate, E. et al.) 313-319 (CIMNE, 2019)

51. Onoda, G. Y. \& Liniger, E. G. Random loose packings of uniform spheres and the dilatancy onset. Phys. Rev. Lett. 64, 2727-2730 (1990).

52. Luding, S. Granular matter: so much for the jamming point. Nat. Phys. 12, 531-532 (2016).

Acknowledgements B.G. and J.B. thank Deutsches Zentrum für Luft- und Raumfahrt (DLR) for continuous support and Deutsche Forschungsgemeinschaft for their support under grant Bl 298/24-2 in the framework of the Research Unit FOR 2285 'Debris disks in planetary systems'. OSIRIS was built by a consortium led by Max-Planck-Institut für Sonnensystemforschung, Göttingen, Germany, in collaboration with CISAS, University of Padova, Italy, Laboratoire d'Astrophysique de Marseille, France, Instituto de Astrofisica de Andalucia, CSIC, Granada, Spain, the Scientific Support Office of the European Space Agency, Noordwijk, The Netherlands, Instituto Nacional de Tecnica Aeroespacial, Madrid, Spain, Universidad Politechnica de Madrid, Spain, the Department of Physics and Astronomy of Uppsala University, Sweden, and Institut für Datentechnik und Kommunikationsnetze der Technischen Universität Braunschweig, Germany. The support of the national funding agencies of Germany (DLR), France (CNES), Italy (ASI), Spain (MEC), Sweden (SNSB), and the ESA Technical Directorate is gratefully acknowledged. Those authors who are part of the VIRTIS and GIADA teams wish to thank the Italian Space Agency (ASI, Italy; contract number I/024/12/2) and Centre National d'Études Spatiales (CNES, France) for supporting their contribution. The contribution of the ROMAP and RPC-MAG teams was financially supported by the German Ministerium für Wirtschaft und Energie and the Deutsches Zentrum für Luft- und Raumfahrt under contract 50QP1401. This research has made use of the scientific software shapeViewer (www.comet-toolbox.com). Video rendering was powered by PRo3D, a viewer for the exploration and analysis of planetary and smaller body surface reconstructions. It was developed by VRVis Zentrum für Virtual Reality und Visualisierung Forschungs-GmbH in close collaboration with Joanneum Research and Imperial College London; see http://pro3d.space for more details. Trajectory and instrumental information relevant to the observations performed on Rosetta was based on the use of SPICE kernels. We acknowledge the important role played by the Rosetta Science Ground Segment, the Rosetta Mission Operations Team and the Philae Lander team(s) in the running of the Rosetta mission and Philae Lander Operations.

Author contributions Identification of the skull-top crevice ice and other touchdown points was made by L.O'R. The lead writer of the paper is L.O'R. Contributions to Methods and sections of Supplementary Information were made by L.O'R., P.H., S.F., 
H.V.H., G.F., A.R., M.C., J.B., B. Gundlach, A.R., M.d.P.C.P. and G.P. The ROMAP and RPC-MAG data analysis was performed by P.H., K.-H.G. and H.-U.A. in close collaboration with L.O'R. The compressive strength and porosity analysis was performed by J.B. and B.G. The OSIRIS image analysis was performed by L.O'R., S.F. and H.V.H. The VIRTIS data analysis was carried out by G.F., A.R., M.C. and F.C. The skull-top crevice ice and dust analysis was carried out by A.R., D.B.-M., M.K., O.G. and N.O. The OSIRIS image processing was performed by S.F., H.V.H., G.K., C.T. and H.S. Trajectory data analysis was performed by B. Grieger, L.O'R., R.A.B., P.H., J.-B.V., C.T. and H.S.

Shape model support and analysis were provided by L.J., J.-B.V., R.A.B., M.d.P.C.P. and G.P. Figures (in support of analysis) were generated by L.O'R., P.H., R.A.B., J.B., B.

Gundlach, S.F., H.V.H., G.F., A.R. and M.C. All authors have participated in the review of the paper and its contents.
Competing interests The authors declare no competing financial interests. One of the authors, N.O., is currently an editor at Nature Communications, but was not in any way involved in the journal review process.

Additional information

Supplementary information is available for this paper at https://doi.org/10.1038/s41586-0202834-3.

Correspondence and requests for materials should be addressed to L.O.

Peer review information Nature thanks Erik Asphaug, Dennis Bodewits, Mathieu Choukroun and the other, anonymous, reviewer(s) for their contribution to the peer review of this work. Peer reviewer reports are available.

Reprints and permissions information is available at http://www.nature.com/reprints. 


\section{Article}

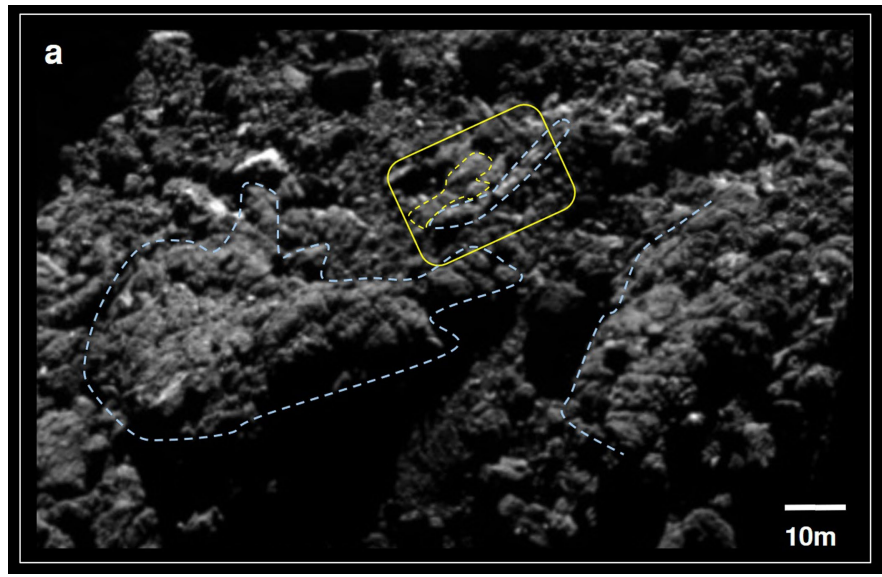

Extended Data Fig. 1 | Comparison of pre-landing and post-landing images. $\mathbf{a}, \mathbf{b}$, Comparison of the location of the skull-top ridge (yellow rectangle) as it looked in a pre-landing OSIRIS image (a; 22 October 2014) and in a post-landing image (b; 14 May 2016). The yellow dashed lines outline the skull-top boulders (see Supplementary Video 1 for a detailed comparison), while context terrain

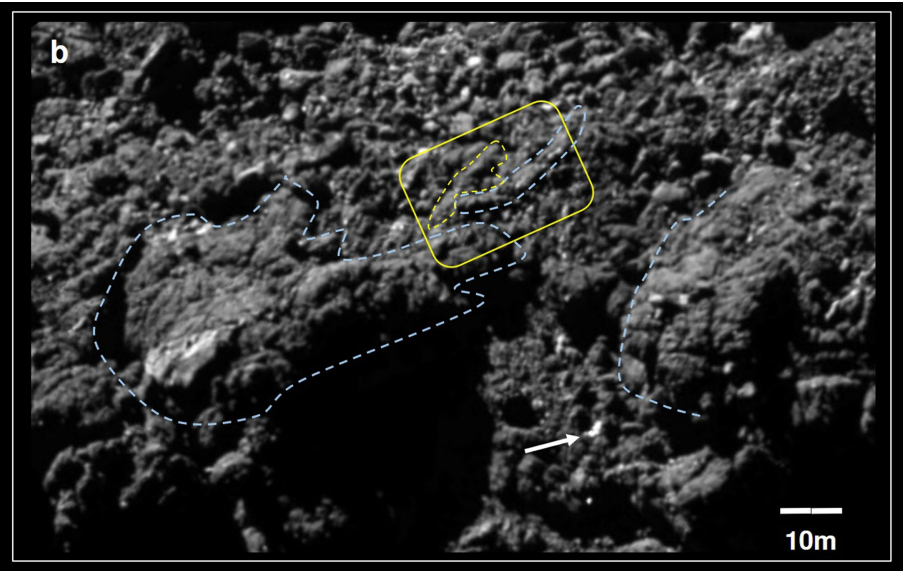

similarities between images are shown in blue dashed lines. Although the spacecraft distance is the same (about $8 \mathrm{~km}$ ), the solar illumination and the Rosetta viewing geometry differ between the images. An arrow points at the Philae lander location in $\mathbf{b}$. 


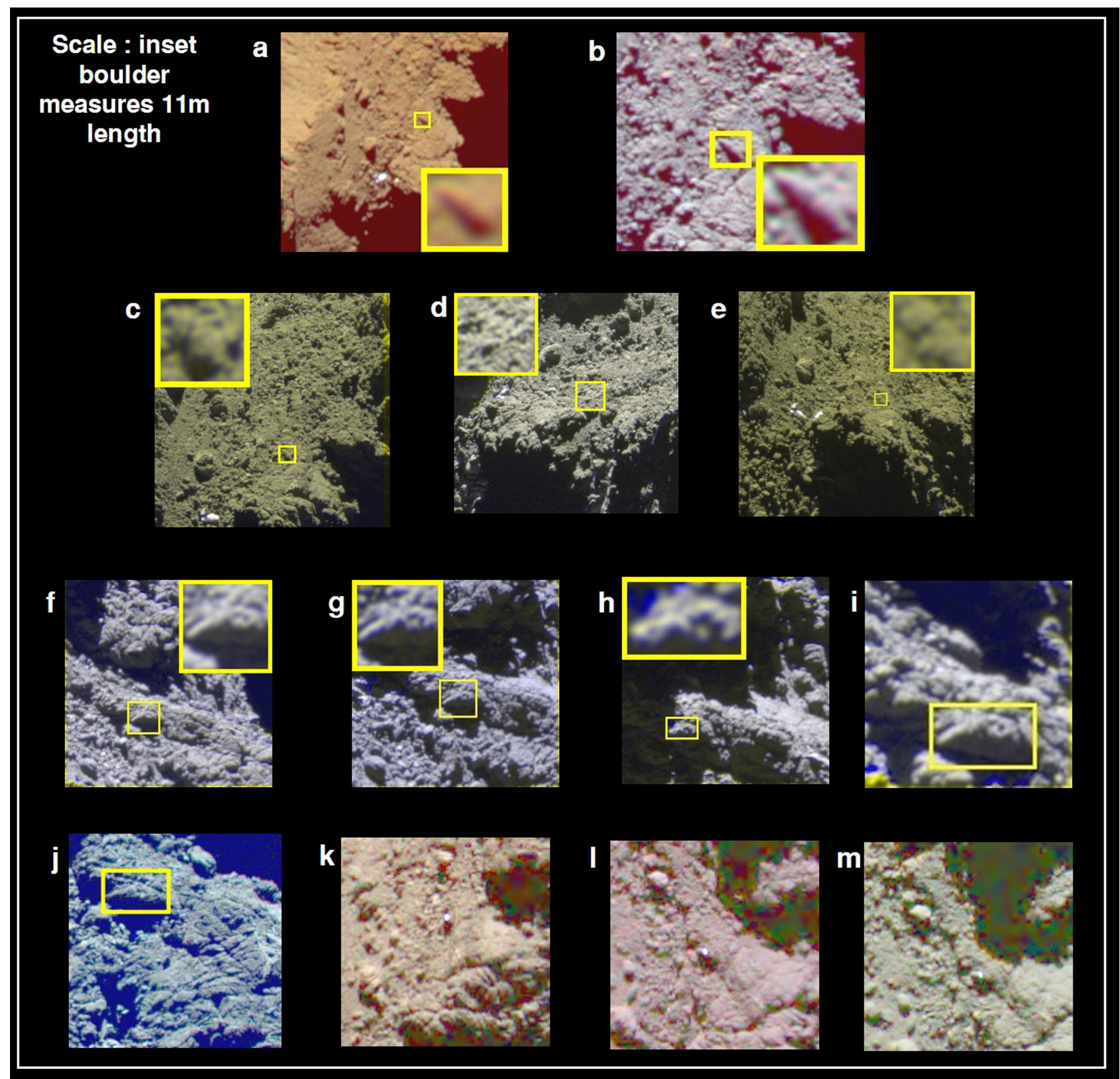

Extended Data Fig. $2 \mid$ OSIRIS colour composites of skull-top boulders. (In $\mathbf{a}-\mathbf{h}$, the boxed feature in the main image is shown magnified in the inset. In $\mathbf{i}, \mathbf{j}$, no magnification is required.) $\mathbf{a}, \mathbf{b}$, Pre-landing images of skull-top boulders. RGB setting; 'green' $=$ F24 $(480.7 \mathrm{~nm})$, 'red' $=$ F22 $(649.2 \mathrm{~nm})$, 'blue' = F16 (360.0 nm). c-e, Skull-top boulders observed in December 2014. RGB setting; 'green' = 'red' = F22 (649.2 nm), 'blue' = F24 (480.7 nm). f-i, The skull-top boulders observed in March 2016. RGB setting: 'green' = 'red' = F22 (649.2 nm), 'blue' = F24 (480.7 nm).j-m, Colour composite of the skull-top boulders in early and mid-June 2016 with the brightly lit ice feature visible in $\mathbf{k}-\mathbf{m}$ specifically. RGB setting; 'green' $=\mathrm{F} 24(480.7 \mathrm{~nm})$, 'red' =F22 (649.2 nm), 'blue' = F16 $(360.0 \mathrm{~nm})$. 


\section{Article}

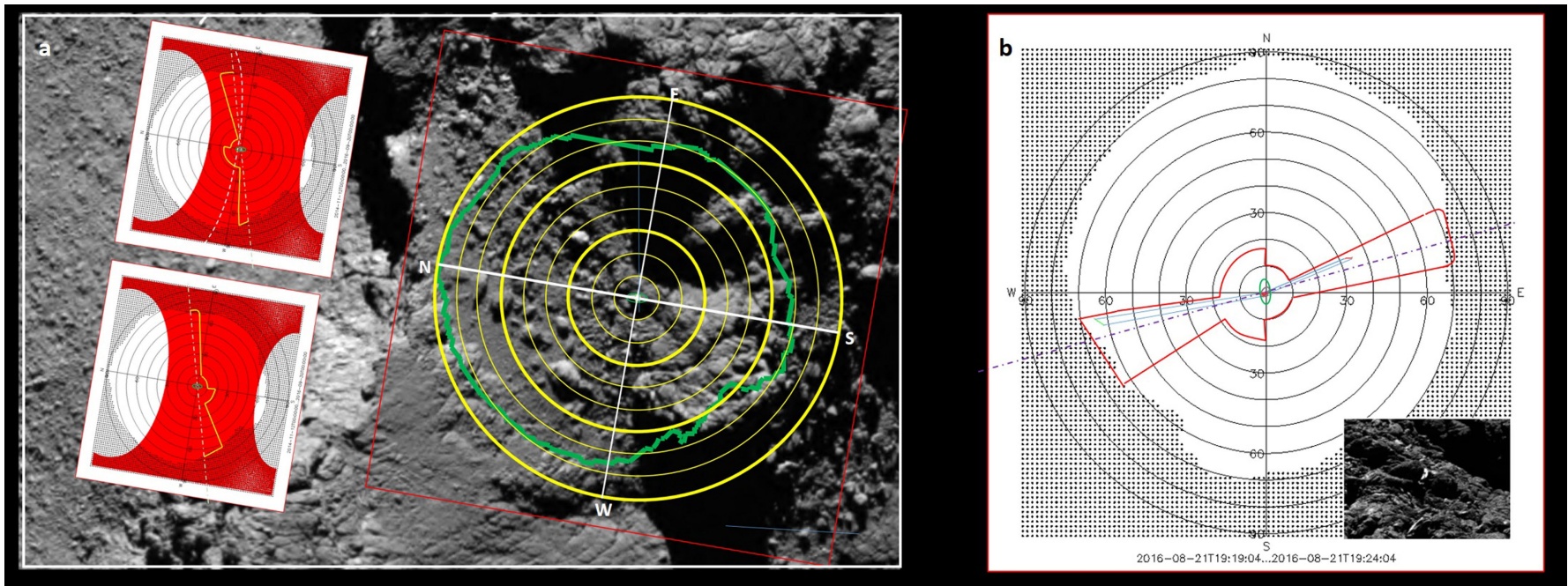

Extended Data Fig. 3 | Illumination geometry of the skull-top crevice. a, OSIRIS NAC image of the Abydos region on 12 June 2016 with three overlaid azimuth plots. The central point of each plot is positioned on the skull-top crevice. The two skull-top boulders are outlined by the green ellipse and the crevice by a tiny purple ellipse inside it. At this central point the viewer is at a $90^{\circ}$ angle, that is, an overhead view; each concentric circle represents a decrease in viewing angle of $10^{\circ}$. The large red box encloses an azimuth plot overlaid on the image and the green line shows the horizon. The red area in the two left-hand azimuth plots shows the track of the Sun over this region during the full period of the Philae landing and to the end of the Rosetta mission, while the yellow line overlay is the crevice's own horizon mask (created as explained in b).b, Example azimuth plot for 21 August 19:19-19:24 UT with Rosetta's position and the Sun's position at this time marked by the very short green and red lines. These short lines are then connected by long blue lines to the central crevice. This gives the line of sight for the OSIRIS camera and for solar illumination of the crevice during this period. We then look at the OSIRIS image (inset) to see if the crevice is illuminated. We find that it is, which is why it lies inside the red-lined horizon mask. The dot-dashed line passes right through the crevice, meaning that the right of that line is the right side of the crevice and the left, the left side. 

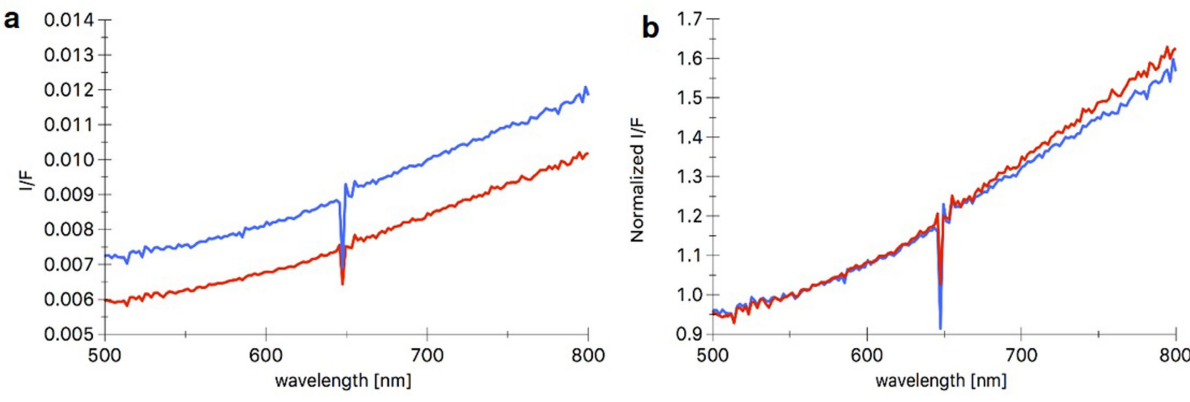

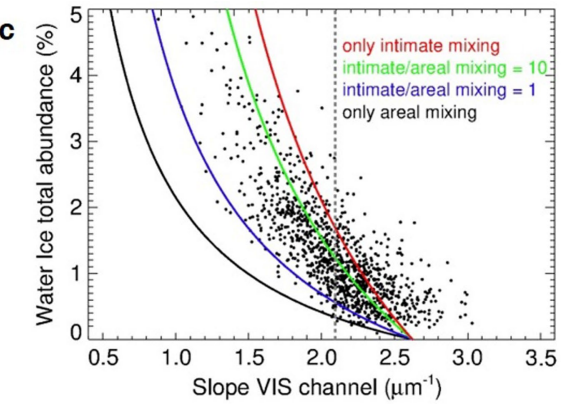

Extended Data Fig. 4 | VIRTIS water-ice analysis plots. a, b, Average radiance factor of the skull-top boulder location (blue curve) and of the nearby dark terrain (red curve) before (a) and after (b) normalization at $550 \mathrm{~nm}$. c, Theoretical abundance of water ice as a function of the spectral slope in the visible (VIS) spectral channel ${ }^{44}$. Black dots and dashed lines indicate a water-ice-rich region observed by VIRTIS to calibrate the theoretical curves.
The black line represents a solely areal mixing case and the red line a solely intimate mixing case. The blue and green lines represent a merge of both, with different mixing ratios of 1 and 10 , respectively. The $x$-axis values correspond to slope values scaled to the dark terrain unit viewing conditions (Fig. 3b, panel b1, red box) for observation V1_00424522185.QUB. 


\section{Article}
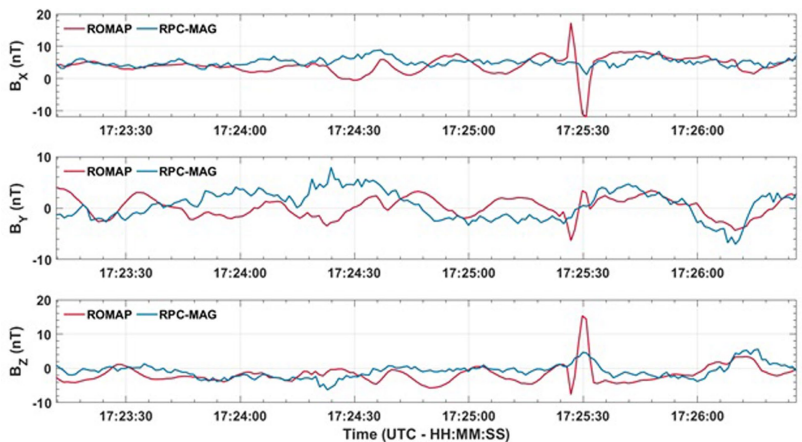

Extended Data Fig. 5 | Combined ROMAP and RPC-MAG magnetic-field and

boom measurements. The three components of the magnetic field observations ( $B_{x}, B_{y}, B_{z}$, respectively top, middle and bottom) are shown starting before TD2 at 17:23:00 UTC and ending shortly after the boom movement was detected. This figure also shows the concurrent orbiter RPC-MAG observations as a reference. See Methods and Supplementary Methods section 7 for a detailed description. 

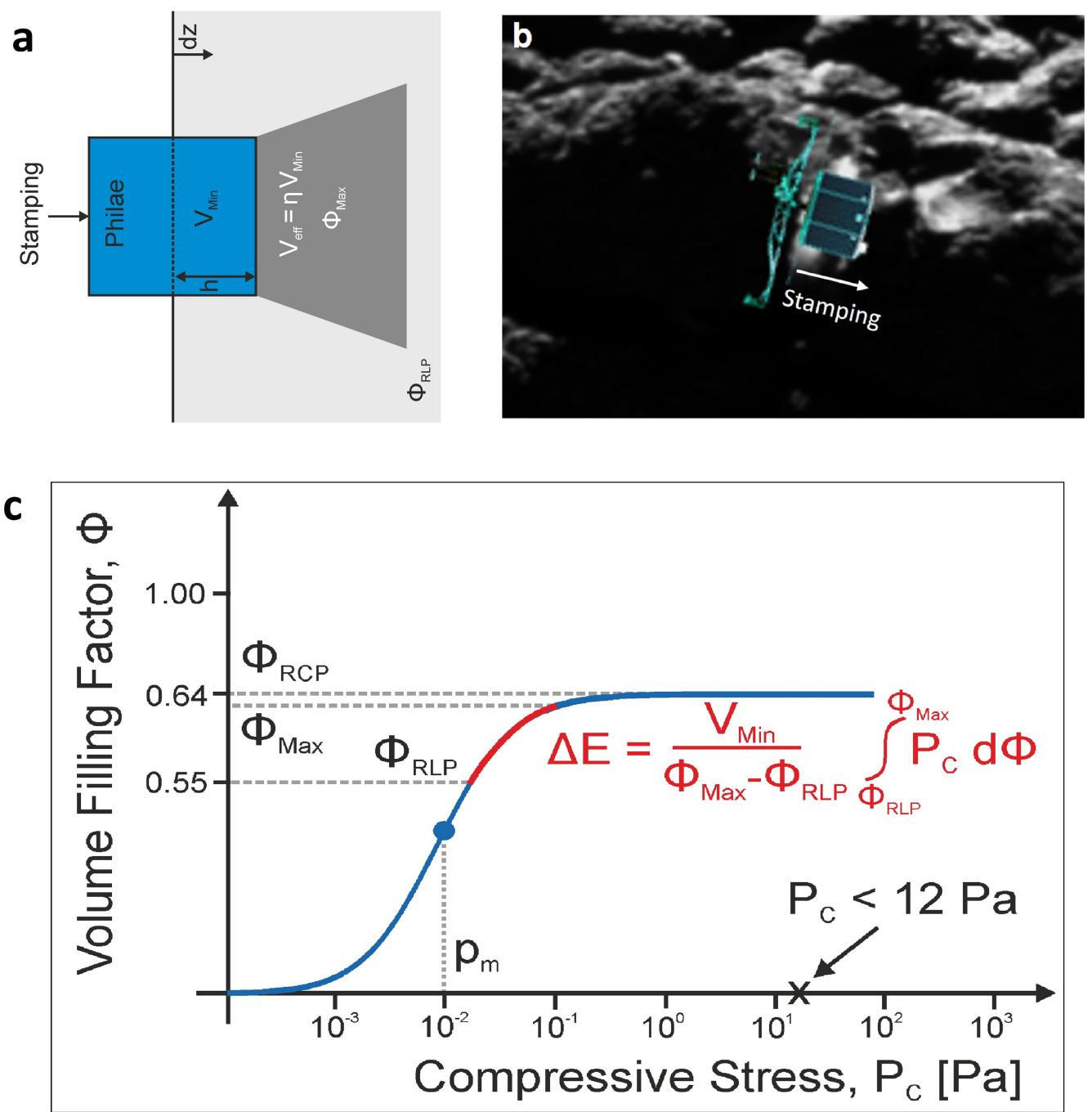

Extended Data Fig. 6 | Philae interaction geometry and boulder volume-filling factor. a, Interaction geometry of Philae with the ice/dust at the moment of deepest penetration in TD2c. b. Philae superimposed on an OSIRIS image (2September 2016), showing an interaction geometry equivalent to that in a.c, Compressive stress curves for a boulder consisting of pebbles. Note that the volume-filling factor $\Phi$ denotes packing of porous pebbles only; these are then further packed to make up the whole boulder. See Methods for a detailed explanation of this panel. 


\section{Article}

Extended Data Table 1 | Estimation of TD2c water-ice content

\begin{tabular}{|c|c|c|c|c|c}
\hline Acquisition time & $\Delta \mathbf{( k m})$ & $\left.\alpha \mathbf{(}^{\circ}\right)$ & Area $\left.\mathbf{( m}^{2}\right)$ & $\rho \mathbf{( 3 0} \boldsymbol{\mu m})$ & $\rho \mathbf{( 1 0 0} \boldsymbol{\mu m})$ \\
\hline 2016-06-12T22:29:58 & 27.5 & 81.4 & 3.7 & $28.8_{-5.3}^{+5.8} \%$ & $30.3_{-5.5}^{+6.1} \%$ \\
\hline 2016-06-14T10:30:32 & 26.7 & 54.0 & 3.5 & $46.4 \%$ & $47.4 \%$ \\
\hline 2016-06-14T22:23:41 & 26.3 & 48.6 & 2.5 & $40.7 \%$ & $43.0 \%$ \\
\hline
\end{tabular}

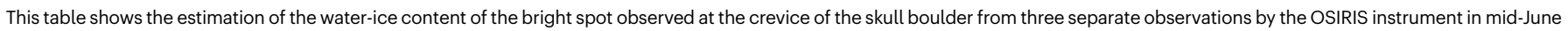

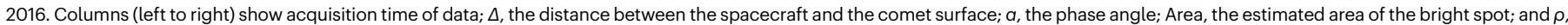

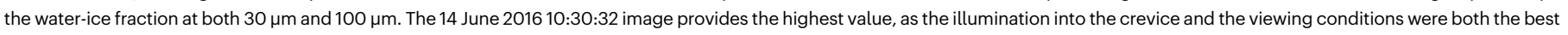
at that time. 
Extended Data Table 2 | Philae lander dynamics for TD2a-d

\begin{tabular}{|c|c|c|c|c|}
\hline & $\begin{array}{c}\text { TD2a } \\
\text { (Initial } \\
\text { Contact) }\end{array}$ & $\begin{array}{c}\text { TD2b } \\
\text { (Dust wall) }\end{array}$ & $\begin{array}{c}\text { TD2c } \\
\text { (Stamping) }\end{array}$ & $\begin{array}{c}\text { TD2d } \\
\text { (Dust compression) }\end{array}$ \\
\hline Start Time (UT) & $17: 23: 48 \pm 10 \mathrm{~s}$ & \multirow{2}{*}{$17: 24: 53 \pm 1 \mathrm{~s}$} & $17: 25: 24 \pm 1 \mathrm{~s}$ & $17: 25: 30 \pm 1 \mathrm{~s}$ \\
\hline End Time (UT) & $17: 24: 22 \pm 1 \mathrm{~s}$ & & $17: 25: 27 \pm 1 \mathrm{~s}$ & $17: 25: 33 \pm 1 \mathrm{~s}$ \\
\hline Contact Time t (s) & $30 \pm 10$ & $2 \pm 1$ & $3 \pm 1$ & $2 \pm 1$ \\
\hline Incoming Kinetic Energy (J) & $2.97 \pm 0.15$ & $1.845 \pm 0.18$ & $1.028 \pm 0.197$ & $0.357 \pm 0.1$ \\
\hline Outgoing Kinetic Energy $(\mathrm{J})$ & $1.974 \pm 0.18$ & $1.028 \pm 0.197$ & $0.357 \pm 0.1$ & $0.10 \pm 0.02$ \\
\hline Energy Loss $(\mathrm{J})$ & $1.342 \pm 0.33$ & $0.817 \pm 0.377$ & $0.671 \pm 0.297$ & $0.257 \pm 0.12$ \\
\hline Incoming Rotation Rate $(\mathrm{mHz})$ & $42.0 \pm 1.0$ & $64.0 \pm 1.0$ & $28 \pm 1.0$ & $33 \pm 1.0$ \\
\hline Outgoing Rotation Rate (mHz) & $64.0 \pm 1.0$ & $28 \pm 1.0$ & $33 \pm 5.0$ & $2 \pm 0.5$ \\
\hline Incoming Vertical Velocity $(\mathrm{m} / \mathrm{s})$ & $\approx-0.194$ & $-0.034 \pm 0.008$ & $-0.017 \pm 0.006$ & $\approx 0$ \\
\hline Incoming Horizontal Velocity $(\mathrm{m} / \mathrm{s})$ & $\approx 0.098$ & $0.109 \pm 0.011$ & $0.125 \pm 0.013$ & $\approx 0$ \\
\hline Outgoing Vertical Velocity $(\mathrm{m} / \mathrm{s})$ & $-0.034 \pm 0.008$ & $-0.017 \pm 0.006$ & $>+0.073$ (after stamping) & $\approx+0.007$ \\
\hline Outgoing Horizontal Velocity $(\mathrm{m} / \mathrm{s})$ & $0.109 \pm 0.011$ & $0.125 \pm 0.013$ & $\approx 0$ & $\approx 0.090$ \\
\hline
\end{tabular}

Shown is a summary of the underlying values and results for TD2, including timing, as described in Methods (section 'Philae lander dynamics at the TD2c point') and Supplementary Methods (section 'ROMAP/RPC-MAG detailed analysis describing Philae's flight through TD2'). Supplementary Video 4 provides an animation showing the dust wall referred to in the table. 